\title{
Carbamazepine toxicity - keep an eye on interaction with clarithromycin
}

\author{
Authors: Waqar Ahmad, Luke Ventress and Satish Artham
}

\section{Introduction}

Carbamazepine is licensed for treatment of convulsions, trigeminal neuralgia and diabetic neuropathy. It is used as an adjunct in alcohol withdrawal and prophylaxis for bipolar disorder. Its plasma concentration is influenced by enzyme CYP3A4 inducers/inhibitors. Clarithromycin, which is a macrolide, inhibits this enzyme resulting in an increased plasma concentration of carbamazepine.

\section{Methods}

We conducted a clinical file consult and extensive literature review on ClinicalKey and Toxbase concerning this topic.

This is a case of a 64-year-old lady with a background history of epilepsy who presented with 3 days history of dizziness which had worsened in the last 24 hours (associated with confusion, slurred speech and unsteadiness on feet). She had recent flulike symptoms and wheeze, and was started on clarithromycin and furosemide by her general practitioner 3 days earlier for likely chest infection and bipedal oedema. She was also taking carbamazepine and levetiracetam for epilepsy.

On examination, she was confused, speech slurred, gait ataxic and Romberg's positive. The rest of the neurological and other system examinations were unremarkable. Relevant investigations included full blood count, urea and electrolytes, blood glucose, serum and urinary osmolality and urinary sodium, thyroidstimulating hormone, morning cortisol level, Synacthen test and serum carbamazepine level, electrocardiogram, computed tomography and head magnetic resonance imaging.

\section{Results and discussion}

Blood results showed a drop in the sodium levels to $124 \mathrm{mmol} / \mathrm{L}$ with a decrease in serum osmolality and increase in urinary sodium and osmolality. Brain imaging and other biochemical tests were unremarkable which ruled out other differential diagnoses like cerebellar stroke, Addison's disease and other causes of hyponatraemia. Furosemide was held initially.

Carbamazepine levels came back very high at $17.6 \mathrm{mg} / \mathrm{L}$ (toxic level). Meanwhile sodium levels dropped further to $119 \mathrm{mmol} / \mathrm{L}$. Carbamazepine and clarithromycin were held resulting in improvement in symptoms and sodium level over the next few days. The carbamazepine level also normalised.

Carbamazepine is a sodium channel blocker with a plasma halflife of 30-40 hours. It is extensively metabolised by cytochrome P450 enzymes, especially CYP3A4. Drugs which inhibit its metabolism resulting in increased levels include macrolides, isoniazid, metronidazole, acetazolamide, diltiazem, verapamil and certain antidepressants. Increased serum levels of carbamazepine can cause symptoms like ataxia, nystagmus, drowsiness, agitation, dilated pupil, respiratory depression, coma and electrolyte disturbances like hyponatraemia and hypokalaemia.

\section{Conclusion}

To conclude, we can say that carbamazepine toxicity can mimic neurological symptoms similar to stroke, so look for an interaction between carbamazepine and clarithromycin. Always use clarithromycin with caution in patients taking carbamazepine.

\section{Conflict of interest statement}

None declared.

\section{References}

1 Albani F, Riva R, Baruzzi A. Clarithromycin-carbamazepine interaction: a case report. Epilepsia 1993;34:161-2.

2 Gélisse P, Hillaire-Buys D, Halaili E et al. [Carbamazepine and clarithromycin: a clinically relevant drug interaction]. Rev Neurol (Paris) 2007;163:1096-9. 